\title{
Short communication: Prediction of retention pay-off using a machine learning algorithm
}

\author{
Saleh Shahinfar, ${ }^{1}$ Afshin S. Kalantari, ${ }^{1}$ Victor Cabrera, ${ }^{2}$ and Kent Weigel \\ Department of Dairy Science, University of Wisconsin, Madison 53706
}

\begin{abstract}
Replacement decisions have a major effect on dairy farm profitability. Dynamic programming (DP) has been widely studied to find the optimal replacement policies in dairy cattle. However, DP models are computationally intensive and might not be practical for daily decision making. Hence, the ability of applying machine learning on a prerun DP model to provide fast and accurate predictions of nonlinear and intercorrelated variables makes it an ideal methodology. Milk class (1 to 5 ), lactation number (1 to 9 ), month in milk (1 to 20 ), and month of pregnancy (0 to 9) were used to describe all cows in a herd in a DP model. Twentyseven scenarios based on all combinations of 3 levels (base, $20 \%$ above, and $20 \%$ below) of milk production, milk price, and replacement cost were solved with the DP model, resulting in a data set of 122,716 records, each with a calculated retention pay-off (RPO). Then, a machine learning model tree algorithm was used to mimic the evaluated RPO with DP. The correlation coefficient factor was used to observe the concordance of RPO evaluated by DP and RPO predicted by the model tree. The obtained correlation coefficient was 0.991, with a corresponding value of 0.11 for relative absolute error. At least 100 instances were required per model constraint, resulting in 204 total equations (models). When these models were used for binary classification of positive and negative RPO, error rates were $1 \%$ false negatives and $9 \%$ false positives. Applying this trained model from simulated data for prediction of RPO for 102 actual replacement records from the University of Wisconsin-Madison dairy herd resulted in a 0.994 correlation with 0.10 relative absolute error rate. Overall results showed that model tree has a potential to be used in conjunction with DP to assist farmers in their replacement decisions.
\end{abstract}

Key words: machine learning, dynamic programming, retention pay-off, dairy cow

Received August 15, 2013

Accepted January 7, 2014.

${ }^{1}$ These authors contributed to this work equally.

${ }^{2}$ Corresponding author: vcabrera@wisc.edu

\section{Short Communication}

Assessing the value of cows in a herd is an important step for making replacement and other critical decisions, such as individual cow breeding or treatment (Cabrera, 2012). Different methods have been developed and used to evaluate cow values in dairy herds, including dynamic stochastic simulation and optimization (Kalantari and Cabrera, 2012). Dynamic programming (DP), which is an optimization technique, has been widely studied over the past several decades (van Arendonk, 1985; De Vries, 2004). Dynamic programming promises the optimal replacement policy, considering several biological and economic factors (Kalantari and Cabrera, 2012), by evaluating the value of keeping or replacing individual cows. These values are used for calculating retention pay-off values (RPO), which can be interpreted as the expected profit from keeping the cow compared with immediate replacement. This value could be used to rank animals and help farmers to make their final replacement decisions (De Vries, 2004). The RPO of a cow changes throughout the lactation, following a curve similar to a lactation curve. Magnitude of RPO is mainly determined by the input parameters of the model, including replacement cost, carcass price, and milk production level of the herd (Kalantari et al., 2010). A DP model can be described by 3 main characteristics: stage length, which is the time between 2 consecutive decisions; state, which is used to describe a cow in the model; and transition probabilities, which are probabilities that are used to weight the values in the model. In the current study, stage length was set at $1 \mathrm{mo}$ and 3 state variables were used to describe the cow in the model. These variables included milk class (1 to 5), lactation number (1 to 9), month in milk (1 to 20), and pregnancy status $[0$ (nonpregnant) or 1 to 9]. Probability of pregnancy, abortion, and involuntary culling were used as transition probabilities. The full details of the DP model and its formulation are explained in Kalantari and Cabrera (2012).

Three different levels of milk production, milk price, and replacement costs, for the base as well as $20 \%$ above and $20 \%$ below the base, were used to create an extensive data set to estimate different ranges of RPO. The total number of records in this data set was 122,716. 
Table 1. Performance of model tree in prediction of retention pay-off with different constraints (minimum numbers of instances per linear model)

\begin{tabular}{lrcc}
\hline $\begin{array}{l}\text { Minimum number of } \\
\text { instances per linear model }\end{array}$ & $\begin{array}{c}\text { Number } \\
\text { of rules }\end{array}$ & $\mathrm{r}$ & $\begin{array}{c}\text { Relative } \\
\text { absolute error (\%) }\end{array}$ \\
\hline 1 & 2,447 & 0.998 & 5.08 \\
$\mathbf{1 0 0}^{1}$ & $\mathbf{2 0 4}$ & $\mathbf{0 . 9 9 1}$ & $\mathbf{1 0 . 9 3}$ \\
200 & 152 & 0.985 & 14.15 \\
400 & 89 & 0.976 & 17.91 \\
800 & 47 & 0.966 & 22.34 \\
1600 & 28 & 0.936 & 30.60 \\
\hline
\end{tabular}

${ }^{1}$ Suggested model constraint and its performance are in bold.

These 3 variables were used because different studies have shown that these variables have the greatest effect on replacement decisions (van Arendonk, 1985; Kalantari et al., 2010). Despite extensive research on DP models and their acknowledged value in finding optimal replacement decisions, this method has not been widely applied for decision-making on commercial farms. One reason could be attributed to the DP complexity, which makes it difficult to both implement and conceptualize (Burt, 1982). Other important reasons could be the time needed for solving DP problems, poor interface with existing farm management programs, and lack of confidence in results. Thus, the ability of model tree (MT) algorithm to provide fast and accurate predic-

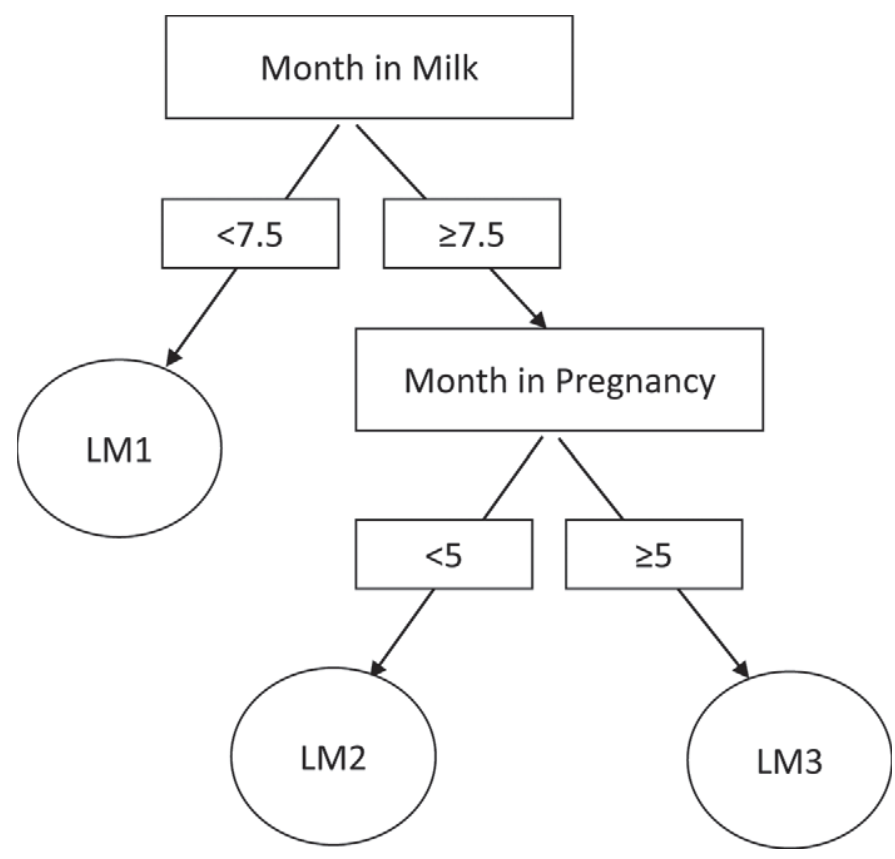

Figure 1. Schematic illustration of the model tree algorithm with 2 explanatory variables (month in milk and month in pregnancy) and 3 linear models in leaves. For example, if month in milk is $<7.5$, the value of retention pay-off (RPO) can be predicted by linear model 1 (LM1), but if it is $>7.5$, then month in pregnancy needs to be considered too. If month in pregnancy is $<5$, linear model 2 (LM2) can be used; if month in pregnancy is $\geq 7.5$, linear model 3 (LM3) will be used. An example of LM2 can be RPO $=0.1025 \times$ month in milk + $2.5118 \times$ month in pregnancy +80.5674 . tions of nonlinear and intercorrelated variables makes it an ideal methodology to be used together with a DP model to mimic the behavior of DP and give a clear interpretation of models and results. Model trees are a type of decision tree used for numeric prediction. They are similar to decision trees because they use a divide and conquer approach to partition the multidimensional state space of the problem (Quinlan, 1992). The MT predicts the values for test instances by the linear model (LM) stored in each leaf based on the attributes of instances that reach that specific leaf (Figure 1). The MT and alternating decision trees have been used in assessment of reproductive performance of dairy herds (Caraviello et al., 2006; Schefers et al., 2010).

In the current study, we developed a user-friendly tool to predict RPO values based on MT, in which the DP remains as a computational core to calculate $\mathrm{RPO}$ whenever the input parameters change. Then, the MT can be retrained based on the new RPO values and used as a terminal predictor of RPO on the farm. Therefore, a Java program was developed using Weka API (Hall et al., 2009) to implement the MT model. An executable version of the program is available online at the University of Wisconsin-Madison Dairy Management web site (www.DairyMGT.info), which can be used to evaluate animals on commercial farms.

The aforementioned data set from the DP model was partitioned randomly, with two-thirds as a training set and one-third as a testing set for MT. Furthermore, to test the trained MT model in a practical situation, 102 records from voluntary culls were extracted from the Allenstein Dairy herd at the University of WisconsinMadison Integrated dairy Facility (Madison, Arlington, and Marshfield, WI), and these were used to perform an independent test for MT.

Relative absolute error was used as the error criterion to assess performance of the MT for two main reasons. First, it measures absolute error, which is not affected by outliers. Second, it considers the relative magnitude of the error compared with the prediction.

$$
\text { Relative absolute error }=\sum_{i=1}^{n} \frac{\left|p_{i}-a_{i}\right|}{\left|a_{i}-\bar{a}\right|},
$$


Table 2. Predicted (model tree) versus actual (dynamic programming) deciles of retention pay-off for 41,723 test cases $^{1}$

\begin{tabular}{lrrrrrrrrrr}
\hline & \multicolumn{10}{c}{ Actual decile } \\
\cline { 2 - 12 } $\begin{array}{l}\text { Predicted } \\
\text { decile }\end{array}$ & \multicolumn{1}{c}{1} & \multicolumn{1}{c}{ 2 } & \multicolumn{1}{c}{3} & \multicolumn{1}{c}{4} & \multicolumn{1}{c}{5} & \multicolumn{1}{c}{6} & 7 & 8 & 9 & 10 \\
\hline 1 & 3,776 & 375 & 21 & 0 & 0 & 0 & 0 & 0 & 0 & 0 \\
2 & 385 & 3,257 & 491 & 34 & 5 & 0 & 0 & 0 & 0 & 0 \\
3 & 10 & 528 & 3,057 & 535 & 30 & 10 & 2 & 0 & 0 & 0 \\
4 & 1 & 12 & 584 & 2,893 & 650 & 31 & 1 & 0 & 0 & 0 \\
5 & 0 & 0 & 18 & 689 & 2,816 & 636 & 11 & 2 & 0 & 0 \\
6 & 0 & 0 & 1 & 20 & 653 & 2,891 & 576 & 30 & 1 & 0 \\
7 & 0 & 0 & 0 & 1 & 17 & 576 & 3,004 & 545 & 29 & 0 \\
8 & 0 & 0 & 0 & 0 & 1 & 23 & 550 & 3,101 & 487 & 10 \\
9 & 0 & 0 & 0 & 0 & 0 & 5 & 27 & 485 & 3,316 & 339 \\
10 & 0 & 0 & 0 & 0 & 0 & 0 & 1 & 9 & 339 & 3,826 \\
\hline
\end{tabular}

${ }^{1}$ Numbers on the diagonal show the instances that model tree predicted the retention pay-off in the same decile as dynamic processing predicted. Above the diagonal show the cases of retention pay-offs that were underestimated by model tree, and below the diagonal are the cases that were overestimated by model tree compared with dynamic processing.

where $p_{i}=$ predicted value; $a_{i}=$ actual value; and $\bar{a}$ is the prediction by an arbitrary predictor, in this case the average of actual values (Witten and Frank, 2005).

In developing a MT, 2 conflicting constraints always exist: number of rules (number of LM stored in the leaves of the tree) and number of instances per LM, which is the number of cases (records) that will reach an arbitrary LM. A trade-off exists between these 2 constraints. Increasing the number of rules is equal to an increase in accuracy of the model on training set but a decrease in number of instances per LM, and therefore loss of generality of the model and poor performance on the new unseen data sets. Hence, finding an optimum point in between these two constraints is critical. Finding this balance is a subjective task and depends on computational limits and acceptable levels of the error. Nevertheless, no systematic way to find such critical balances exists, which usually depends on an expert's knowledge. Performance of MT in terms of number of rules, correlation coefficient, and relative absolute error, with respect to the minimum numbers of instances per linear model as an indicating constraint, are shown in Table 1. A noticeable trend was observed in which increasing the minimum numbers of instances per linear model resulted in a decrease in the number of rules and correlation coefficient, but an increase in the error criterion. Predicted (MT) versus actual (DP) RPO for corresponding 6 scenarios from Table 1 are graphically presented in Figure 2.

Considering the results from Table 1 , it seems that a minimum of 100 instances per LM would be a logical constraint because the number of rules dropped considerably, to one-tenth in comparison with one instance per model (2,447 vs. 204), and, at the same time, the correlation coefficient and relative absolute error did not change substantially. Actual and predicted RPO were divided into 10 equal intervals called deciles. Dis- tributions of predicted versus actual deciles of RPO are presented in Table 2. Numbers on the diagonal show the instances that MT predicted the RPO in the same decile as DP. Numbers above the diagonal show the cases of RPO that were cases of RPO underestimated by MT, and numbers below the diagonal are the cases that were overestimated. In general, the MT resulted in $76.5 \%$ correctly predicted RPO, $12 \%$ overestimated RPO, and $11.5 \%$ underestimated RPO. Inside each decile, variation was also relatively low. For example, within the lower decile, which included the negative values, the actual and predicted RPO distributions were $-107.4 \pm 56.6$ and $-105.6 \pm 59.5$, respectively.

As the sign of RPO is more important than its magnitude (Groenendaal et al., 2004), a binary evaluation of RPO prediction by MT was considered. The confusion matrix in the case of binary classification of $\mathrm{RPO}$ is presented in Table 3. Classification of RPO into positive and negative cases showed an error rate of $1 \%$ false negative (FN) cases and $9 \%$ false positive (FP) cases, which indicates the advantage of the method in identifying negative cases. Here, FN are the terminal mistakes in which cows that are predicted to have a negative RPO are culled from the herd erroneously, whereas FP are mistakes that can be corrected by identifying the animal as a cull in the next replacement decision round. However, the cost of FN and FP will depend on the magnitude of the RPO in which if a

Table 3. Confusion matrix for percentage of binary prediction of retention pay-off for 41,723 test cases

\begin{tabular}{lcc}
\hline & \multicolumn{2}{c}{ Actual (dynamic programming) } \\
\cline { 2 - 3 } $\begin{array}{l}\text { Predicted } \\
\text { model tree) }\end{array}$ & Positive & Negative \\
\hline Positive & 0.91 & 0.09 \\
Negative & 0.01 & 0.99 \\
\hline
\end{tabular}


1
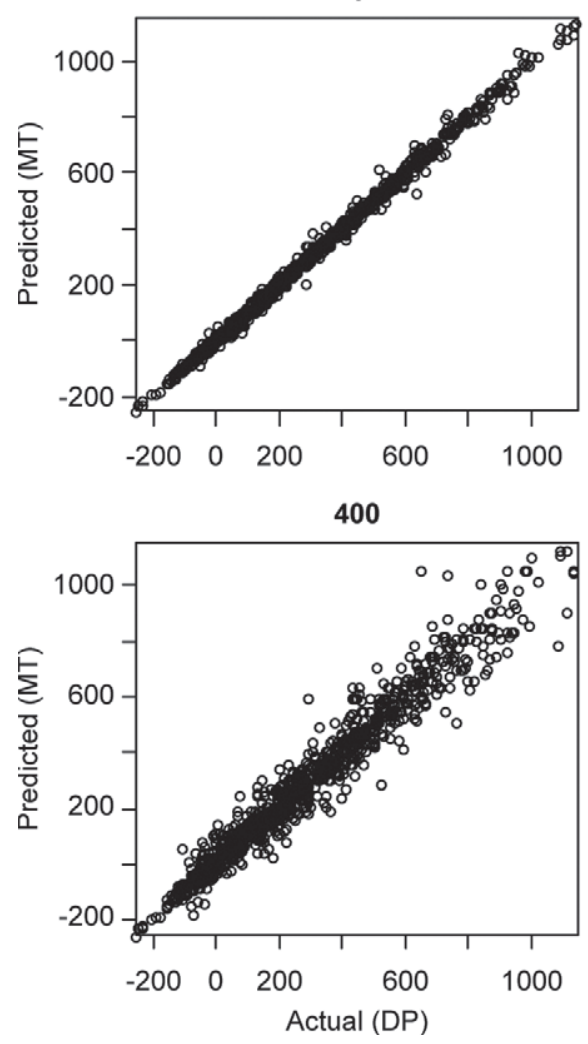

100
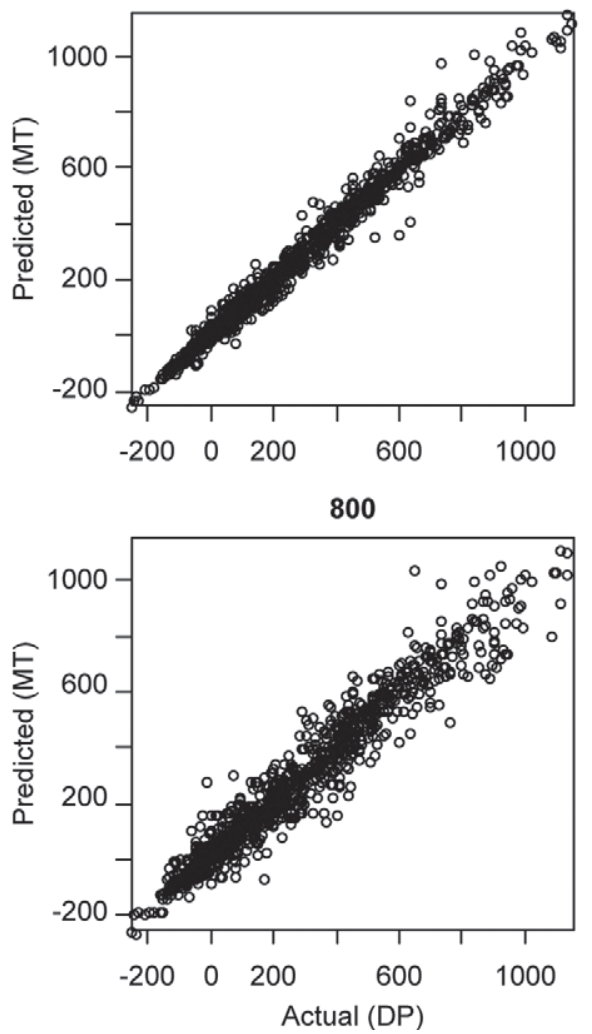

200
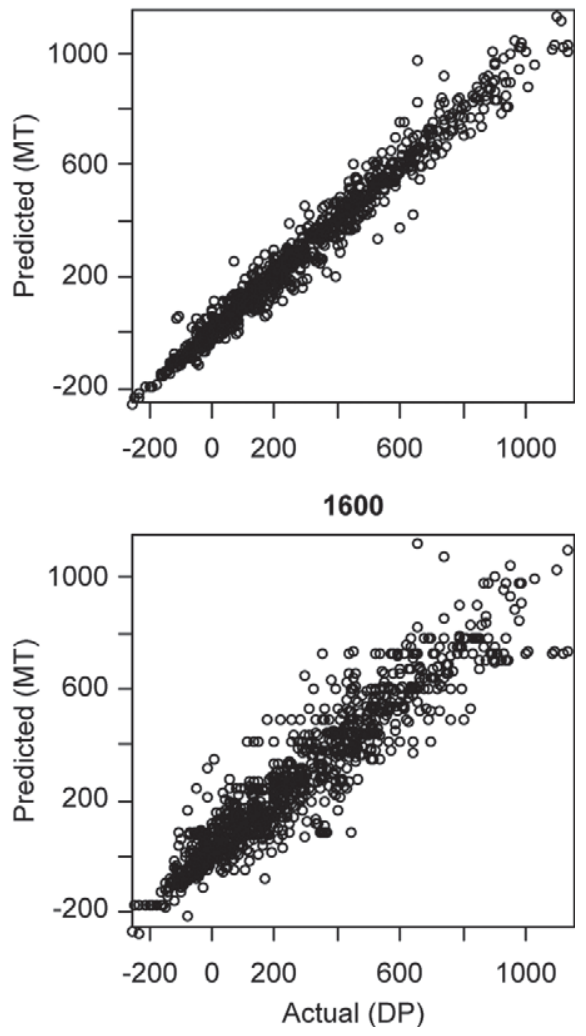

Figure 2. Actual (predicted by dynamic programming; DP) versus predicted (predicted by machine learning model tree; MT) values of retention pay-off for 6 different scenarios with the test set shown in Table 1.

specific FN case has a positive RPO just a little above the zero the related cost would be zero and vice versa.

The RPO prediction using MT is an efficient method for assessing the value of keeping or culling specific cows and helping farmers make economical culling decisions. A correlation coefficient of 0.994 with a 0.10 relative absolute error rate was obtained between the trained model and the observed records of 102 actual voluntary culling events from the University of WisconsinMadison dairy herd, which indicates its accuracy and relevance in real-life replacement decisions.

\section{ACKNOWLEDGMENTS}

This project was supported by Agriculture and Food Research Initiative Competitive Grant no. 2010-8512220612 from the USDA National Institute of Food and Agriculture (Washington, DC).

\section{REFERENCES}

Burt, O. R. 1982. Dynamic programming: Has its day arrived? West. J. Agric. Econ. 7:381-394.

Cabrera, V. E. 2012. A simple formulation and solution to the replacement problem: A practical tool to assess the economic cow value, the value of a new pregnancy, and the cost of a pregnancy loss. J. Dairy Sci. 95:4683-4698.
Caraviello, D. Z., K. A. Weigel, M. Craven, D. Gianola, N. B. Cook, K. V. Nordlund, P. M. Fricke, and M. C. Wiltbank. 2006. Analysis of reproductive performance of lactating cows on large dairy farms using machine learning algorithms. J. Dairy Sci. 89:4703-4722.

De Vries, A. 2004. Economics of delayed replacement when cow performance is seasonal. J. Dairy Sci. 87:2947-2958.

Groenendaal, H., D. T. Galligan, and H. A. Mulder. 2004. An economic spreadsheet model to determine optimal breeding and replacement decisions for dairy cattle. J. Dairy Sci. 87:2146-2157.

Hall, M., E. Frank, G. Holmes, B. Pfahringer, P. Reutemann, and I. H. Witten. 2009. The WEKA data mining software: An update. SIGKDD Explor. 11:10-18.

Kalantari, A. S., and V. E. Cabrera. 2012. The effect of reproductive performance on the dairy cattle herd value assessed by integrating a daily dynamic programming model with a daily Markov chain model. J. Dairy Sci. 95:6160-6170.

Kalantari, A. S., H. Mehrabani-Yeganeh, M. Moradi, A. H. Sanders, and A. De Vries. 2010. Determining the optimum replacement policy for Holstein dairy herds in Iran. J. Dairy Sci. 93:2262-2270.

Quinlan, J. R. 1992. Learning with continuous classes. Pages 343-348 in Proc. 5th Aust. Joint Conf. Artif. Intell. World Scientific, Singapore.

Schefers, J. M., K. A. Weigel, C. L. Rawson, N. R. Zwald, and N. B. Cook. 2010. Management practices associated with conception rate and service rate of lactating Holstein cows in large, commercial dairy herds. J. Dairy Sci. 93:1459-1467.

van Arendonk, J. A. M. 1985. Studies on the replacement policies in dairy cattle. II. Optimum policy and influence of changes in production and prices. Livest. Prod. Sci. 13:101-121.

Witten, I. H., and E. Frank. 2005. Data Mining, 2nd ed. Elsevier, San Francisco, CA. 